\title{
RENTABILIDAD Y PRECIO DE LA TIERRA AGRÍCOLA: UN ESTUDIO SOBRE LA REGIÓN PAMPEANA ARGENTINA ${ }^{1}$
}

\author{
PROFITABILITY AND PRICE OF \\ AGRICULTURAL LAND: A STUDY ON THE \\ PAMPEAN REGION IN ARGENTINA
}

\author{
Silvina B. Marcolini Tulliani' ${ }^{2}$ Carmen S. Verón Medina ${ }^{3}$ y Marisa D. Goytia García ${ }^{4}$
}

\section{RESUMEN}

La revisión de la literatura relacionada con los precios de la tierra y sus determinantes incluye estudios que explican la evolución del precio a partir de la rentabilidad del recurso y la capacidad de generación de flujos de fondos y otros que lo hacen a través de sus atributos o características. El presente estudio vincula la evolución del precio de la tierra agrícola, con la rentabilidad del recurso.

El objetivo del trabajo es analizar si el cambio en el valor de la tierra del área más productiva de la zona pampeana durante la década comprendida entre los años 2005-2014, depende o tiene una relación directa con la rentabilidad generada por el recurso. Se plantean para ello, sus posibles destinos alternativos, producción o cesión en arrendamiento y a su vez se considera para la producción, soja de primera y de segunda, maíz y trigo. Para medir la rentabilidad de la tierra de acuerdo a sus usos alternativos se utilizan magnitudes contables de acuerdo al destino.

El trabajo relaciona el precio de la tierra como variable dependiente con los márgenes brutos de los cultivos de soja de primera, soja de segunda, maíz y trigo y el valor del arrendamiento, como variables independientes. Se trabaja con un análisis de regresión múltiple que evidencia que es la decisión de producción la que incide sobre el precio de la tierra.

Los resultados obtenidos demuestran que si bien la producción de soja de primera, soja de segunda, maíz y trigo contribuye a explicar el precio de la tierra, la soja de primera, es la variable de mayor incidencia sobre el mismo.

Palabras claves: Margen bruto, precio, rentabilidad, tierra agrícola.

Recepción: 12/12/2015. Aprobación: 19/01//2016.

\section{ABSTRACT}

The review of land prices literature and its determinants includes studies that explain the evolution of the price from the resource profitability and ability to generate cash flows or their attributes and characteristics. Our study links the evolution of agricultural land price, with the profitability of the resource.

The aim of the paper is to analyze whether the change in the value of pampean land during the

\footnotetext{
1 Artículo de investigación científica y tecnológica. Proyecto "La información contable de las actividades agropecuarias regionales: usuarios y contenido", Universidad Nacional de Rosario, Argentina, período 2014-2017.

2 Silvina B. Marcolini Tulliani, Facultad de Ciencias Económicas y Estadística de la Universidad Nacional de Rosario, Argentina, smarcoli@fcecon.unr. edu.ar

3 Carmen S. Verón Medina, Facultad de Ciencias Económicas y Estadística de la Universidad Nacional de Rosario, Argentina, cveron@fcecon.unr.edu.ar 4 Marisa D. Goytia García, Facultad de Ciencias Económicas y Estadística de la Universidad Nacional de Rosario, Argentina, mgoytia@fcecon.unr. edu.ar
} 
decade between 2005-2014, depends on or is directly related to the profitability generated by the resource. We propose two alternative destinations, leasing or production (soybean, corn and wheat). We use accounting aggregates in anytime.

The work relates the land price as dependent variable and soybean, corn and wheat gross margins and lease value, as independent variables. It works with multiple regression analysis which shows that production is the decision which affects land price.

The results show that while soybean production, corn and wheat help to explain the land price, soybean is the variable with greatest impact on it.

Keywords: Agricultural land, Gross margin, price, profitability.

\section{INTRODUCCIÓN}

\subsection{Descripción del problema}

El factor tierra es indispensable para el desarrollo de la actividad agropecuaria y es un recurso finito e inmóvil. Su expansión, está limitada por las fronteras terrestres y marítimas y su utilización está condicionada por el clima y las condiciones ecológicas de la región. En Argentina durante los últimos años, el valor monetario de la tierra destinada a la agricultura, ha experimentado cambios positivos sustanciales. A su vez, la rentabilidad de la tierra depende principalmente de su calidad y ubicación. El extenso territorio argentino, de norte a sur y de este a oeste tiene suelos de variada calidad y diferente ubicación. Resulta por lo tanto imposible referirse al factor tierra considerando a la superficie del país en su conjunto.

Para el trabajo, se ha seleccionado del territorio argentino, la zona delimitada por el sur de la provincia de Santa Fe y norte de la provincia de Buenos Aires, por tratarse de la región agrícola más productiva del país. Esta región es conocida como el corazón de la pampa, tiene suelos fértiles, clima templado, regímenes de lluvia adecuados y está cercana a las unidades de embarque portuarias desde donde se exporta la producción. Además, la zona elegida es la más importante del país en lo referido a la exportación de granos y subproductos, representando en promedio el $70 \%$ de la exportación física total. En ella, el principal cultivo es la soja, y le siguen en orden de importancia el maíz y el trigo. Asimismo, el cambio del precio de la tierra ha generado que muchas inversiones se destinen a su adquisición, para su posterior cesión en arrendamiento. La creciente demanda de tierras para destinar a producción, frente a una oferta cada vez más concentrada del recurso en manos de inversores, determinó un crecimiento importante en los valores de dichos arrendamientos.

El objetivo del trabajo es analizar si el cambio en el valor de la tierra del corazón de la zona pampeana argentina, durante la década comprendida entre los años 2005-2014, depende o tiene una relación directa con la rentabilidad generada por el recurso. Se plantean para ello, sus posibles destinos alternativos, producción o cesión en arrendamiento y a su vez se considera para la producción, soja de primera y de segunda, maíz y trigo. Los resultados indican que la producción de soja de primera es la que mayor incidencia tiene en el precio de la tierra.

A partir de esta introducción en el apartado siguiente se presenta un análisis del sector agrícola argentino, luego la revisión de la literatura, para continuar con el diseño de la investigación, metodología y resultados y finalizar con las conclusiones.

\subsection{El sector agrícola argentino en cifras}

El sector agropecuario ha sido en el siglo pasado el pilar del desarrollo económico de Argentina. Su trascendencia ha ido declinando a través del tiempo y su contribución al Producto Bruto Interno (PBI) disminuyendo. Durante la década 2005-2014, se ha convertido nuevamente en el sostén de la economía de Argentina, a través de la producción de soja. Diversos factores tales como, el aumento de la superficie destinada a su cultivo, los mayores rindes obtenidos en zonas marginales provenientes de la aplicación de nuevas tecnologías, la expansión de la frontera agropecuaria y los mejores precios internacionales, convirtieron a la soja y sus derivados en el principal producto agropecuario nacional.

Se justifica esta afirmación a partir de datos obtenidos de estadísticas nacionales. Para ello, se revisa la participación del sector agropecuario en el producto bruto interno, la participación de los complejos oleaginosos y cerealeros en el total de exportaciones y la evolución de la producción de cereales y oleaginosas.

Respecto a la participación del sector agropecuario en el producto bruto interno, y según 
datos suministrados por el Centro de Economía Internacional (CEI), del Ministerio de Relaciones Exteriores y Culto de la República Argentina, en el año 2013 la participación del sector agropecuario en el PBI es del 5,9\%, mientras que la industria participa el $13,2 \%$ y el sector de servicios el $54,7 \%$. Dicha participación ha ido disminuyendo, pasando del 7,9\% en el año 2005 al 5,9\% en el 2013. Sin embargo, si bien el productor primario es el primer eslabón en la cadena agroalimentaria, no opera solo, sino que se integra en un conjunto complejo de actividades que engloba a las industrias que venden al sector agropecuario, el sector agropecuario, las industrias transformadoras de los productos procedentes y el sector de distribución, que pone a disposición del consumidor los diversos alimentos generados. Por lo expuesto la vinculación del sector primario con el PBI no puede ser analizada aisladamente.

Si se revisa la participación del sector agropecuario en las exportaciones, se advierte que, a través de la actividad agrícola, Argentina obtiene la principal fuente de ingreso de divisas. Para publicar los referentes productivos que originan las principales exportaciones del país, el Instituto Nacional de Estadística y Censos (INDEC) adopta la denominación de complejos exportadores ya que, casi la totalidad de las posiciones arancelarias que son representativas en el total de exportaciones, no lo son por sí mismas, sino que su importancia se evidencia cuando se las articula entre sí a partir del marco conceptual que brindan los eslabonamientos productivos.

Como se puede apreciar en la Tabla I, la participación del complejo oleaginoso desde el año 2009 al 2013, presenta un incremento que va desde el 25 al $27 \%$ sobre el total de complejos exportadores. Por su parte los complejos cerealeros tienen una participación que oscila entre el $7 \%$ y el $13 \%$; lo que determina un total entre los dos de 32 a $37 \%$.

Tabla I: Complejos oleaginosos y cerealeros 2009-2013.

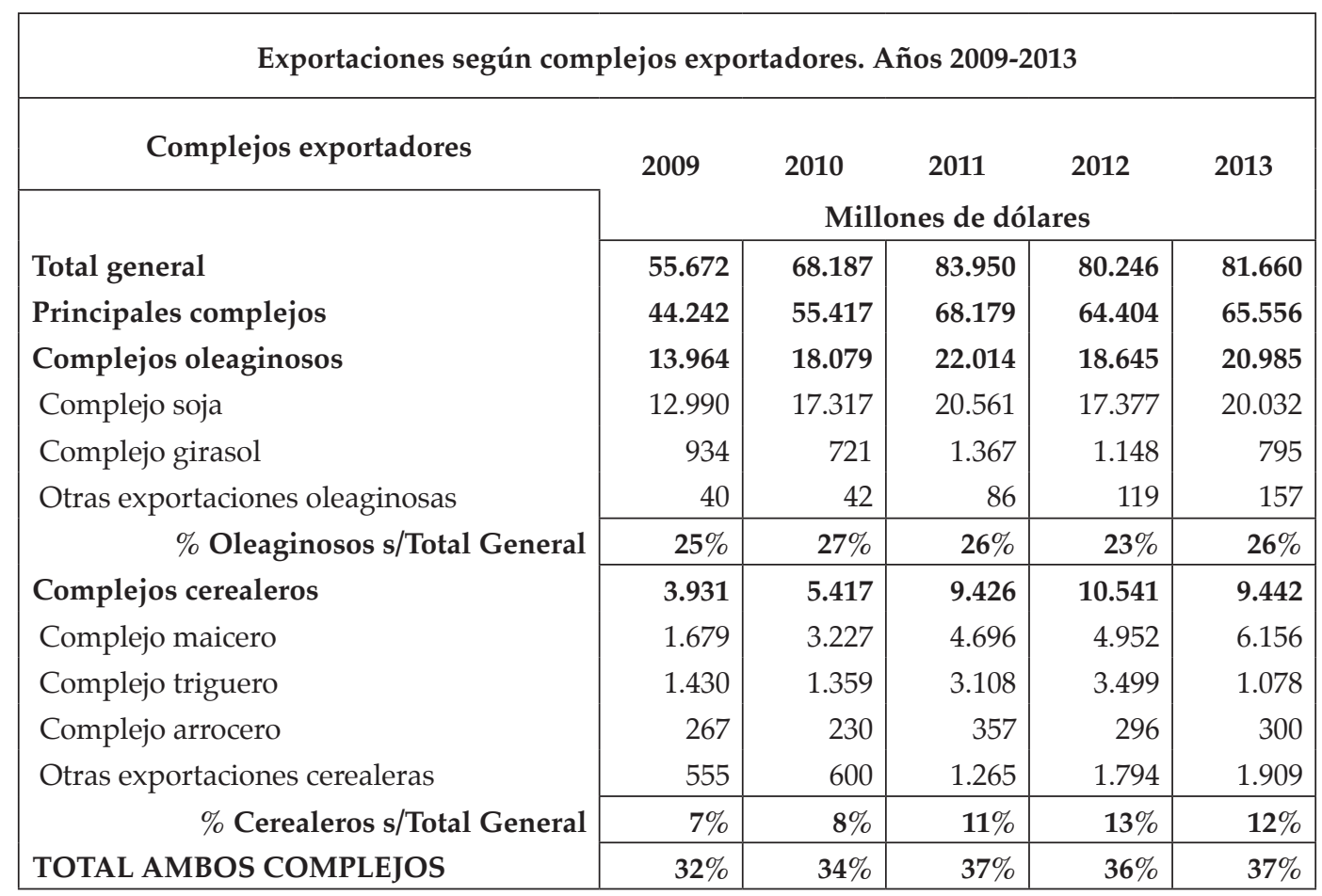

Fuente: INDEC

El complejo soja representa dentro de oleaginosas un total que oscila entre el 93 y $96 \%$. Por su parte, dentro del complejo cerealero, el trigo evidencia una clara disminución desde el año 2009 al 2013 con una participación que varió del 26 al $11 \%$. El maíz, en cambio, aumenta pasando del 43\% en el año 2009, al 65\% en el 2013.

Respecto a la evolución de los cultivos selec- 
cionados se revisan las campañas 2008/2009 a $2012 / 2013$, en base a datos oficiales del INDEC. Se considera la producción de los cultivos sobre los que se basa el trabajo en relación a la producción total de cereales y oleaginosas. Se advierte que, si bien la participación en el total de cereales, del maíz y el trigo, ha disminuído del 81 al 79\%, el trigo tuvo una clara disminución que se contrapone a un incremento importante del maíz. La soja, que representa más del $90 \%$ de la producción de oleaginosas ha aumentado su participación en los períodos considerados.

La información sobre participación en las exportaciones del complejo soja y sobre la evolución de la producción de soja, trigo y maíz evidencian la relevancia que los tres cultivos mencionados tienen dentro de la producción agrícola de Argentina. La variación de sus precios y de los niveles de producción podrían ser factores determinantes del incremento del valor de la tierra destinada a su cultivo.

\subsection{Revisión de la literatura}

En términos económicos, la forma más utilizada para determinar el valor de un bien, es la estimación del valor presente del flujo de ingresos netos que generará. Esto sugiere que dicho valor aumentará en la medida que el flujo neto esperado de ingresos también lo haga y viceversa.

El Informe Final (2009), publicado por el Departamento de Economía Agraria de la Pontificia Universidad Católica de Chile, explica claramente que la literatura relacionada con los precios de la tierra y sus determinantes se clasifica en:

- Estudios que explican la evolución del precio de la tierra a partir del flujo de fondos generado por la actividad

- Estudios que explican los factores determinantes del precio de la tierra a través de sus atributos, también llamados estudios hedónicos.

Dentro de la primera línea de investigación se ubican Troncoso y Tobar (2005), Tobar Cabello (2003), Alston (1986), Chavas y Tomas (1999) y Lanteri (2012). Dentro de la segunda línea de investigación se ubican Fuentes y Bravo-Ureta (2003), Gracia et al. (2004), Troncoso et al. (2010), Donoso et al. (2013) y, Stewart y Libby (1998).

El estudio de Troncoso y Tobar (2005), calcula el ingreso neto generado por la producción agrícola, a partir del valor de mercado de la pro- ducción proveniente de la tierra, neto del valor de los factores empleados para obtener dicha producción. Este planteo, claramente económico, vincula el valor del bien con la rentabilidad que puede generar y para medirla calcula los términos de Intercambio de la agricultura.

Tobar Cabello (2003), para obtener el índice de precios real logrado por los agricultores utiliza los principales productos agropecuarios. Los resultados indican que los cambios en la rentabilidad de la agricultura influyen parcialmente en el precio de la tierra, siendo el subsector vitivinícola el de mayor impacto.

Alston (1986), plantea como variables explicativas del crecimiento del precio de la tierra agrícola en Estados Unidos, durante la década del 70, al aumento de los ingresos netos generados por ésta y a las expectativas inflacionarias. Sin embargo, a partir de los resultados de un trabajo empírico sugiere que la mayor parte del crecimiento real del precio de la tierra se explica por el incremento de los ingresos netos generados por ésta, mientras que las expectativas inflacionarias han tenido un efecto negativo sobre dicho precio, aunque relativamente pequeño.

Chavas y Thomas (1999), trabajan con evidencia de Estados Unidos para los períodos 1950-1996, a través del modelo CAPM. Los resultados indican que, la aversión al riesgo y los costos de transacción tienen efectos significativos sobre el precio de la tierra.

Por su parte, Lanteri (2012), explica que el incremento en los precios de la tierra, podría vincularse con las cotizaciones de las materias primas exportables, el crecimiento de la economía y las mejoras en los términos de intercambio con el exterior. Plantea como variables explicativas, los precios internacionales reales de las materias primas agropecuarias, el producto bruto interno real, el índice general de precios, la tasa de interés real y el tipo de cambio real. Trabaja con datos anuales en una serie temporal que abarca los períodos 1960-2012 y lo hace en Argentina y Estados Unidos. Los resultados sugieren que el producto interno bruto real sería el factor explicativo más relevante en Argentina mientras que, la tasa de interés real sería el factor más importante en Estados Unidos. El autor concluye pero no demuestra, que en Argentina, el crecimiento de la economía habría provocado que los excedentes generados por los sectores industria y servicios se vuelquen a la adquisición de tierras, y sea el incremento de la demanda del recurso el factor más importante del cambio de precios operado en los períodos de crecimiento.

Por otro lado, dentro de los denominados 
estudios hedónicos se encuentran Bravo-Ureta y Fuentes (2003), que explican el valor por hectárea en dólares de la tierra a partir de lo que clasifican como variables internas (calidad del suelo, disponibilidad de riego y presencia de infraestructura en la finca) y variables espaciales (distancia de la finca a la carretera o ciudad más cercana y zona donde se ubica la finca). Se refieren a predios agrícolas de determinadas regiones chilenas durante los períodos 1981-1996. Los resultados indican que tanto las variables internas como las espaciales impactan significativamente en el valor del suelo.

Sanjuán et al. (2004), explican los precios de la tierra en la provincia de Zaragoza, España, a partir de sus características agronómicas, ambientales y sociales. Encuentran una asociación poco significativa con los factores socioeconómicos y sugieren que el principal factor determinante de los precios es la orientación productiva de las tierras.

En un artículo posterior, Troncoso et al. (2010), analiza la influencia de los atributos físicos de la tierra como determinantes de su precio. En este caso, se revisaron todas las ventas de establecimientos ocurridas en una provincia determinada de Chile durante los períodos 2003-2006. Las variables independientes son el tamaño del establecimiento en hectáreas, la calidad de la tierra clasificada en clases, las lluvias medidas en litros por segundo, la conectividad como distancia a la ruta asfaltada más cercana y la ubicación clasificada por zonas. Los resultados demuestran que todas las variables son significativas y la ubicación resulta ser la más importante.

En la misma línea, Donoso et al. (2013), comparan los factores determinantes del valor de la tierra durante dos períodos diferentes (19781998 y 1999-2008). Consideran como variables explicativas del valor de la tierra, a factores productivos y no productivos. Los primeros se convierten en variables dicotómicas que asumen valor 1 si la tierra es apta para alguna de las alternativas de producción, fruticultura, forestación, cosecha de granos, o ganadería y valor 0 si no lo es. Los factores no productivos se refieren a la evolución en el tiempo del precio por hectárea, superficie, existencia de construcciones o mejoras, distancia a la ciudad capital regional y nacional y precio del producto agrícola más importante de la región. Los resultados indican que los factores determinantes del precio de la tierra han cambiado significativamente en los dos períodos analizados. Otro estudio de Donoso et al. (2013) que abarca los períodos 1998-2008 en Chile, rescata como variables explicativas del precio de la tierra agrícola, su idoneidad para la producción y para diferentes cultivos, su ubicación, los costos de transporte y las posibilidades de uso residencial. Sugiere que el valor por hectárea disminuye cuando el número de hectáreas ofrecidas aumenta.

Asimismo, Stewart y Libby (1998), agregan como determinante del valor de la tierra a la incidencia de las políticas públicas en el área agrícola. El estudio empírico se desarrolla en Estados Unidos durante los períodos 1990-1995.

El presente estudio se ubica dentro de la revisión de la literatura en los que vinculan la evolución del precio de la tierra con los flujos generados por ésta. La medición de los flujos se determina a partir de la rentabilidad del recurso, a través de magnitudes contables que se explican en el apartado siguiente.

\section{DISEÑO DE LA INVESTIGACIÓN Y METODOLOGÍA}

\subsection{Diseño de la investigación}

La rentabilidad del recurso tierra está determinada por varias cuestiones pero, en cualquiera de los casos y tratándose de productos agrícolas, utilizando como unidad de costeo, o unidad en torno a la que se agrupan los costos, la hectárea. Esto implica que tanto los ingresos como los costos se trabajan por hectárea, que representa la unidad de medición más importante y utilizada en la producción agrícola.

Entre los determinantes de la rentabilidad de la tierra se menciona en primer término, la decisión respecto a su forma de explotación. En este aspecto puede optarse por llevar adelante la producción o cederla en arrendamiento. La opción de llevar adelante el proceso, lleva consigo la decisión de producción, que consiste en definir el producto a sembrar. Los cultivos más habituales de la zona son soja, maíz y trigo, aunque otras producciones alternativas pueden ser posible. El flujo estará dado por los ingresos generados por la producción medidos en quintales por hectárea y a su vez, multiplicados por el precio del grano a la fecha de la medición, menos todos los costos y gastos necesarios para llevar adelante el proceso, calculados de la misma forma.

En la actividad agrícola es muy utilizada por los profesionales de las ciencias agronómicas para determinar el resultado de una empresa agropecuaria, la técnica del margen bruto. Se trata de una magnitud obtenida de la contabilidad de gestión. El margen bruto es el resultado 
de restar al ingreso bruto (Rendimiento promedio de la producción en quintales por hectárea, por precio de venta del producto), los gastos directos totales de producción y comercialización tanto fijos como variables. Los gastos directos en términos generales son los relacionados con la preparación de la tierra, siembra, mantenimiento del cultivo y cosecha, transporte (fletes corto y largo), acondicionamiento y almacenamiento del grano.

Por su parte, el arrendamiento refleja el precio pagado por un tercero por el uso y goce de la tierra, a su propietario. Generalmente en la zona analizada, se fija en quintales de soja por hectárea. En ese caso, el flujo generado por la tierra estará dado por el valor de dicho arrendamiento que es igual a la cantidad de quintales de soja multiplicados por el precio del grano, a la fecha de la medición.

El margen bruto resulta un flujo comparable al arrendamiento ya que en cualquiera de los casos no se consideran los costos de estructura o indirectos y se miden en pesos por hectárea.

Para el análisis se tomaron los períodos 20052014. La elección surge de la observación de los cambios producidos en el precio de la tierra durante los años considerados. Además, el extenso territorio argentino tiene tierras y climas de los más variados, por lo que no resulta posible generalizar el análisis, y en virtud de lo expuesto se ha seleccionado la zona cubierta por el sur de la provincia de Santa Fe y norte de la provincia de Buenos Aires.

Los datos relevados corresponden a los cultivos de soja de primera y de segunda, maíz y trigo, obteniendo para cada período los precios en dólares por tonelada de los mismos, los márgenes brutos de cada uno, y el valor del arrendamiento, también expresado en dólares por hec- tárea. Además se buscó el precio de la tierra, en dólares por hectárea.

Resulta importante aclarar que, todas las variables monetarias han sido expresadas en dólares estadounidenses por dos razones, en primer término para neutralizar el efecto inflacionario y el deterioro del poder adquisitivo que sufre la moneda argentina respecto a otras, y en segundo término porque los precios del grano y sus correspondientes costos se fijan en dólares aunque a posteriori se traducen a moneda local.

Todos los datos utilizados se obtienen de la revista Márgenes Agropecuarios de cada uno de los meses que componen los años 2005-2014. Se trabaja en consecuencia con diez años y doce meses por año, lo que determina un total de ciento veinte observaciones. Para el cálculo del margen bruto la revista considera mensualmente y para cada cultivo dos escenarios de rendimiento, un promedio bajo y un promedio alto. En todos los casos se utiliza el promedio alto determinado en cada período mensual.

\subsection{Metodología}

De acuerdo a lo expuesto y con el objetivo de analizar si los posibles usos alternativos de la tierra inciden en el precio de la misma, se plantea un modelo de regresión múltiple, en el que la variable dependiente es el precio de la tierra para el período $t$, y las variables independientes son el valor del arrendamiento y los márgenes brutos del cultivo de soja de primera, soja de segunda, maíz y trigo para el mismo período. Se espera averiguar en qué medida la variable dependiente puede estar explicada por las variables independientes.

La ecuación del modelo a desarrollar es la siguiente:

$$
P T_{t}=\beta_{0}+\beta_{1} M B S 1_{t}+\beta_{2} M B S 2_{t}+\beta_{3} M B M_{t}+\beta_{4} M B T_{t}+\beta_{5} V A+\in
$$

Donde:

$P T_{1}=$ Precio de la tierra en el período $t$ (en dólares por hectárea)

$M B S 1_{t}=$ Margen bruto soja de primera en el período $t$ (en dólares por hectárea)

$M B S 2_{\mathrm{t}}=$ Margen bruto soja de segunda en el período $t$ (en dólares por hectárea)
$M B M_{t}=$ Margen bruto maíz en el período $t$ (en dólares por hectárea)

$M B T_{t}=$ Margen bruto trigo en el período $t$ (en dólares por hectárea)

$V A_{\mathrm{t}}=$ Valor del arrendamiento en el período $t$ (en dólares por hectárea)

Se espera que los $\beta$ en todos los casos resul- 
ten positivos y demuestren en consecuencia, la asociación entre el precio de la tierra y las variables independientes identificadas en este análisis. Asimismo se espera que $\operatorname{los} \beta_{1}, \beta_{2}, \beta_{3}, \beta_{4}, \beta_{5}$ sean significativos y que el valor del $\beta_{1}$ sea superior a los anteriores. Esto evidenciaría que la decisión de producción más rentable es la del cultivo de soja de primera por sobre la posibilidad de arrendar la tierra a terceros o destinarla a producir otros cultivos. Sin embargo se espera que los resultados de $\operatorname{los} \beta_{2}, \beta_{3} \mathrm{y} \beta_{4}$, sean inferiores al $\beta_{5}$, lo que indicaría que la producción de soja de segunda, trigo y maíz no sólo es menos rentable que la producción de soja de primera, sino también que la cesión del predio rural en arrendamiento. A su vez, se pueden considerar individualmente, por un lado los resultados del $\beta_{3}$ y por el otro las sumatoria de $\operatorname{los} \beta_{2}$ y $\beta_{4}$ ya que la siembra de soja de segunda va acompañada de la siembra de trigo, constituyendo otra alternativa de producción. Existen expectativas respecto a los resultados de esta comparación.

\section{RESULTADOS}

En la Tabla II, se presentan los resultados de los estadísticos descriptivos de cada una de las variables consideradas:

Tabla II: Estadísticos descriptivos de las variables.

\begin{tabular}{|c|c|c|c|c|c|}
\hline & N & Mínimo & Máximo & Media & Desviación estándar \\
\hline PT & 120 & 5400 & 17500 & 12001,67 & 3798,27 \\
\hline MBS1 & 120 & 234,10 & 864,40 & 521,08 & 140,76 \\
\hline MBS2 & 120 & 113,90 & 593,40 & 263,33 & 78,54 \\
\hline MBM & 120 & 115,80 & 1009,10 & 522,64 & 202,52 \\
\hline MBT & 120 & $-68,30$ & 492,00 & 225,90 & 120,03 \\
\hline VA & 120 & 207,00 & 688,00 & 434,24 & 121,97 \\
\hline
\end{tabular}

Para el análisis estadístico, se aplica a cada una de las variables el test de Kolmogorov para identificar si se comportan como una normal y definir en consecuencia el test estadístico a aplicar. Los resultados de la prueba arrojaron que todas las variables excepto el VA, se distribuyen como una normal.

Se plantea entonces una regresión lineal múltiple aplicando un procedimiento de regresión por pasos. Se ha tomado esta decisión a partir del comportamiento diferente de las variables independientes, el número de variables seleccionadas para explicar el modelo, y la ausencia de trabajos previos que sean orientativos respecto a la elección de las variables relevantes. Por lo expuesto, el control de las variables que integran la ecuación de regresión se basa en criterios estadísticos. La selección de las variables en este caso, se basa en un criterio de significación, incorporando al modelo, aquellas variables que contribuyen de manera significativa a su ajuste. Esto determina que las variables independientes ingresan al sistema por orden de relevancia. Bajo este procedimiento, el modelo excluye la variable VA por lo que los resultados que se exponen en la Tabla III responden a la siguiente ecuación:

$$
P T_{t}=\beta_{0}+\beta_{1} M B S 1_{t}+\beta_{2} M B S 2_{t}+\beta_{3} M B M_{t}+\beta_{4} M B T_{t}+€
$$

Tabla III: Resultados R cuadrado.

\begin{tabular}{|c|c|c|c|c|}
\hline Modelo & $\mathbf{R}$ & R cuadrado & R cuadrado ajustado & Error estándar \\
\hline $\mathbf{1}$ & 0,728 & 0,530 & 0,526 & 2614,166 \\
\hline $\mathbf{2}$ & 0,908 & 0,824 & 0,821 & 1605,665 \\
\hline $\mathbf{3}$ & 0,923 & 0,852 & 0,848 & 1480,250 \\
\hline $\mathbf{4}$ & 0,927 & 0,860 & 0,855 & 1444,517 \\
\hline
\end{tabular}


Este procedimiento, genera resultados para 4 modelos, el número 1 considera como variable independiente al MBS1, el número 2 agrega MBS2, el número 3 incluye MBT y el número 4 incorpora MBM, por lo que para analizar los resultados de las cuatro variables independientes de la ecuación debemos interpretar el renglón correspondiente al modelo 4 . Se advierte que los resultados del $\mathrm{R}$ cuadrado indican que las cuatro variables consideradas en su conjunto explican el $86 \%$ de la varianza de la variable dependiente.

La tabla IV, incluye los resultados del ANOVA y se transcribe la parte pertinente al modelo 4 , es decir correspondiente a las cuatro variables independientes.

Tabla IV: Anova.

\begin{tabular}{|c|c|c|c|c|c|}
\hline Modelo & Suma de cuadrados & gl & Media cuadrática & F & Sig. \\
\hline Regresión & 1476837403 & 4 & 369209350,7 & 176,941 & 0,000 \\
Residuo & 239962263,8 & 115 & 2086628,381 & & \\
Total & 1716799667 & 119 & & & \\
\hline
\end{tabular}

El valor del estadístico $\mathrm{F}$ y del nivel crítico Sig. indican que existe relación lineal significativa entre la variable dependiente y las independientes y evidencian un buen ajuste del modelo.
Finalmente, la tabla V siguiente, incluye los resultados del coeficiente estandarizado Beta y la prueba $\mathrm{t}$

Tabla V: Coeficiente estandarizado Beta.

\begin{tabular}{|c|c|c|c|c|}
\hline Modelo & $\begin{array}{c}\text { Coeficiente no } \\
\text { estandarizado B }\end{array}$ & $\begin{array}{c}\text { Coeficiente estandarizado } \\
\text { Beta }\end{array}$ & $\mathbf{t}$ & Sig. \\
\hline Constante & $-577,691$ & 2,996 & $-1,027$ & 0,307 \\
MBS1 & 80,842 & $-2,059$ & 20,337 & 0,000 \\
MBS2 & $-99,563$ & $-0,178$ & $-14,870$ & 0,000 \\
MBT & $-5,625$ & $-0,210$ & $-4,385$ & 0,000 \\
MBM & $-3,935$ & & $-2,610$ & 0,010 \\
\hline
\end{tabular}

El coeficiente no estandarizado B resulta positivo para la variable MBS1 y negativo para las restantes. El cambio de signo puede indicar un alto grado de asociación entre alguna de las variables independientes. Su interpretación debe tomarse con cautela ya que el valor de cada uno se ajusta teniendo en cuenta la presencia del resto de las variables.

Sin embargo, es claro lo que evidencia el resultado del coeficiente estandarizado Beta que es comparable entre sí y proporciona una idea sobre la importancia relativa de cada una de las variables independientes en la ecuación. En este caso la variable de mayor peso en la ecuación es MBS1, le siguen MBS2, MBM y MBT. Es importante aclarar que los resultados de este coeficien- te deben interpretarse en valores absolutos. Se advierte además que la variable MBS1 considerada individualmente tiene mayor incidencia en la variable dependiente que las variables MBS2 y MBT consideradas conjuntamente.

Por su parte la prueba t nos muestra qué variables son relevantes en la ecuación de regresión. En el caso analizado, las cuatro variables poseen coeficientes significativos distintos de 0 , por lo que todas contribuyen en parte a explicar lo que ocurre con la variable dependiente, pero vuelve a ser clara la mayor incidencia de la variable MBS1. Esto es coherente con los resultados de los niveles críticos Sig. que en todos los casos resulta inferior a 0,05 . 


\section{CONCLUSIÓN}

El cambio en el valor de la tierra de la zona que se denomina, el corazón de la zona pampeana argentina, durante la década comprendida entre los años 2005-2014, depende de la rentabilidad generada por el recurso. Si bien dicha rentabilidad puede analizarse a partir de dos destinos alternativos, cesión en arrendamiento y producción de granos, los resultados obtenidos demuestran que es este último el que incide en la variable dependiente.

Además, la producción de granos ha sido analizada a partir de los márgenes brutos de los cultivos más importantes de la región. Tanto la producción de soja de primera, soja de segunda, maíz y trigo contribuyen a explicar el precio de la tierra, pero los resultados demuestran que la soja de primera, es la variable de mayor incidencia sobre el mismo.

Este estudio contribuye a la revisión de la literatura en dos aspectos. En primer término al utilizar una alternativa diferente para la determinación de la rentabilidad de la tierra obtenida a partir de la contabilidad de gestión, como es el margen bruto. En segundo término porque extiende los análisis realizados sobre el tema, por otros autores, a Argentina.

Finalmente, se considera que existe al menos una limitación. Si bien el dólar constituye una moneda de uso generalizado para todas las operaciones relacionadas con la actividad agropecuaria en Argentina, no constituye la moneda de curso legal en el país. La conversión a pesos argentinos de cada una de las variables utilizadas en este estudio genera diferencias en los resultados, que no son atribuibles al análisis realizado sino a cuestiones de política económica nacional.

\section{BIBLIOGRAFÍA}

Alston, J. (1986). An analysis of growth of U.S. farmland prices 1963-1982. American Journal of Agricultural Economics, 68, 1-9.

Bravo-Ureta, B., \& Fuentes, P. (2003). Determinantes del valor de la tierra agrícola en Chile. In Selected Paper presented at the VIII Congress of Chilean Agricultural Economists, Santiago, Chile. Organized by the Asociación de Economistas Agrarios de Chile.

Centro de Economía Internacional. Ministerio de Relaciones Exteriores y Culto de la República
Argentina (2015). Recuperado el 12 de julio de 2015, http:// www.cei.gob.ar.

Chavas, J. \& Thomas, A. (1999). A Dynamic Analysis of Land Prices. American Agricultural Economics Association, 81, 772-784.

Donoso, G., Cancino, J., Olguín, R., \& Schönhaut, D. (2013). A comparison of farmland value determinants in Chile between 1978-1998 and 1999-2008.Ciencia e Investigación Agraria, 40(1), 85-96.

Donoso, G., Cancino, J., \& Foster, W. (2013). Farmland values and agricultural growth: The case of Chile. Economía agraria y recursos naturales, 13(2), 33-52.

Garrido Fernández, A. (1993). Papel de la Biotecnología en el Sistema Agroalimentario: Situación, Presente y Perspectivas Futuras, La Agricultura del siglo XXI, Mundi Prensa, 189.

Goytia, M. \& Marcolini, S. (2013). Cultivo de soja: Inflación y tipo de cambio, presentado en las XV jornadas Nacionales de la Empresa Agropecuaria, Tandil, Argentina.

Lanteri, L. (2013). Comportamiento de los precios reales de la tierra agrícola en la Argentina y en los Estados Unidos, 1960-2012. Atlantic Review of Economics: Revista Atlántica de Economía, 2(1), 5-22.

Lazzati, N. \& Pacheco, J. (2004). Análisis de la Evolución del Componente Estacional del Precio de la Soja en Argentina. Implicancias para el Productor Agropecuario. XXXIX Reunión Anual AAEP. Buenos Aires.

Marcolini, S. \& Goytia, M. (2008). Una alternativa de financiamiento del sector agropecuario que contribuye a su desarrollo, presentado en el Congreso Internacional de Competitividad y Desarrollo, Universidad de Santo Tomás, Bucaramanga, Colombia.

Márgenes Agropecuarios (2005-2014), Argentina, http:/ / www.margenes.com.

Ministerio de Economía y Producción, Datos recuperados el 15 de julio de 2015, http: / / www. mecon.gob.ar.

Paruelo, J. M., Guerschman, J. P., Piñeiro, G., 
Jobbagy, E. G., Verón, S. R., Baldi, G., \& Baeza, S. (2006). Cambios en el uso de la tierra en Argentina y Uruguay: marcos conceptuales para su análisis. agrociencia, 10(2), 47-61.

Rudi, E. (2011). Gestión de empresas del sector primario - Conceptos teóricos, Manuscrito no publicado, utilizado en la carrera de posgrado Especialización en Costos y Gestión Empresarial, Facultad de Ciencias Económicas y Estadística, Universidad Nacional de Rosario.

Sanjuán, A. I., Hurlé, J. B., Pérez, L., \& Royo, A. G. (2004). Análisis hedónico de los precios de la tierra en la provincia de Zaragoza. Revista española de estudios agrosociales y pesqueros, (202), 5170.

Sili, M. \& Soumoulou, L. (2011). La problemática de la tierra en Argentina. Conflictos y dinámicas de uso, tenencia y concentración. Fondo Internacional de Desarrollo Agrícola. Ministerio de Agricultura, Ganadería y Pesca, agosto.

Stewart, P. \& Libby, L. (1998). Determinants of farmland value: the case of DeKalb County, Illinois. Review of Agricultural Economics, 20(1), 8095.

Tobar, A. \& Osvaldo, P. (2003). Evolución del precio de la tierra y de la rentabilidad de la agricultura chilena entre el período 1983 y 2002. Memoria de Título, Facultad de Ciencias Agrarias, Universidad de Talca, Talca.

Troncoso, J., Aguirre, M., Manriquez, P., Labarra, V., \& Ormazábal, Y. (2010). Influence of physical attributes on the price of land: the case of the Province of Talca, Chile. Ciencia e investigación agraria, 37, 105-112.

Troncoso, J. \& Tobar, P. (2005). Evolución de la rentabilidad de la agricultura y del precio de la tierra, periodo 1983-2002. Panorama Socioeconómico, (30), 0.

UC, F. A. (2009). Valor de la tierra agrícola y sus factores determinantes. 